\title{
Weighted Two-Band Target Entropy \\ Minimization for the Reconstruction of Pure \\ Component Mass Spectra: Simulation Studies and the Application to Real Systems
}

\author{
Huajun Zhang and Marc Garland \\ Department of Chemical and Environmental Engineering, National University of Singapore, Singapore, \\ Singapore
}

Yingzhi Zeng and Ping Wu

Institute of High Performance Computing, Singapore, Singapore

\begin{abstract}
A method is proposed, on the basis of a recently developed algorithm-Band Target Entropy Minimization (BTEM) - to reconstruct mass spectra of pure components from mixture spectra. This method is particular useful in dealing with spectral data with discrete features (like mass spectra). Compared to the original BTEM, which has been applied to differentiable spectroscopies such as Fourier-transfer infrared spectroscopy (FTIR), ultraviolet (UV), Raman, and nuclear magnetic resonance (NMR), the latest modifications were obtained through: (1) Reformulating the objective function using the peak heights instead of their derivatives; (2) weighting the abstract vector $V^{T}$ to reduce the effect of noise; (3) using a two-peak targeting strategy (tBTEM) to deal with strongly overlapping peaks; and (4) using exhaustive search to locate all the component spectra. A set of 50 multi-component mass spectra was generated from ten reference experimental pure component spectra. Many of the compounds chosen have common MS fragments and therefore, many of the pure component spectra have considerable intensity in same data channels. In addition, a set of MS spectra from a real system with four components was used to examine the newly developed algorithm. Successful reconstruction of the ten component spectra of the simulated system and the four component spectra of the real system was rapidly achieved using the new tBTEM algorithm. The advantages of the new algorithm and its implication for rapid system identification of unknown mixtures are readily apparent. (J Am Soc Mass Spectrom 2003, 14, 1295-1305) (C) 2003 American Society for Mass Spectrometry
\end{abstract}

$\mathrm{I}$ n the chemical sciences, one constant challenge is to identify pure components from mixtures and/or evolving reactive systems. Usually, one attempts to separate/purify the components before analysis, but such efforts are often very time consuming and sometimes totally ineffective. Many advanced combined analytical instruments, e.g., GC-MS, HPLC-MS, and HPLC-NMR, have been used to perform separation, obtain pure component spectra, and finally positively identify of the pure components present. However, there exist many situations where separations are difficult or even impossible (i.e., reactive systems with transient or labile species). Accordingly, considerable effort has been invested in the reconstruction of pure component spectra from mixture data alone without

Published online September 13, 2003

Address reprint requests to Dr. Marc Garland, Department of Chemical and Environmental Engineering, National University of Singapore, 4 Engineering Drive 4, Singapore 117576, Singapore. E-mail: chemvg@nus.edu.sg separation and without relying on prior information. Considerable progress has been made recently in the interpretation of continuously differentiable spectroscopic data, such as FTIR, Raman, and UV-VIS. The present contribution presents a method for recovering discrete pure component mass spectra from mixture data, based on a recently developed algorithm, bandtarget entropy minimization (BTEM).

Over the past few decades, pure component spectral reconstruction from mixture data has received considerable attention [1, 2]. Many of these methods are based on some sort of algebraic projection method e.g., SIMPLSMA [3, 4], IPCA [5], OPA-ALS [6], but these methods frequently fail with mixtures containing more than 3-4 components, and/or with features that are highly overlapping. Furthermore, they are unable to recover minor components.

One of the more interesting and different approaches, proposed by Sasaki et al [7], involves entropy minimization. Indeed, entropy minimization is known 
to be a powerful pattern recognition tool [8] and is associated with the principle of simplicity [9]. Recently entropy minimization has gained renewed interest, [10-13] and a new BTEM [14] method has been developed to recover all observable components from an unknown mixture, including trace components that are difficult to identify with other algorithms. It has been successfully applied to real experimental systems with more than 10 species present-particularly transition metal homogeneous chemical syntheses [15-18]. These efforts have been successful in identifying pure component spectra from FTIR and Raman [19] data where the spectroscopic measurements are continuous functions.

Mass spectral data presents new challenges as well as new opportunities. A few researchers have reported approaches to reconstruct pure component spectra from mixtures. Feng and Liang [20] proposed an approach to retrieve components' mass spectra. The procedure first checks the weighted reference to determine the presence of the reference spectra in a mixture and then uses a non-negative least squares regression to find the contributions of the components in the mixture. Phalp et al. [21] reported a modified SIMPLISMA approach (TSIMPLISMA) that used the concept of "representative spectra" of MS. Instead of using the pure-variables in the SIMPLISMA, TSIMPLISMA defines and evaluates the purity of the spectra. The spectrum with the highest purity value represents the "representative" spectrum for a component and the contributions from this component are removed from the data set. The procedure proceeds sequentially for the remaining components. The incorporation of expert knowledge also contributes to the usefulness of TSIMPLISMA. In summary, the identification of pure component mass spectra from mixture spectra usually requires some sort of prior information concerning the reference spectra or expert knowledge.

It should be reiterated that the BTEM approach is considerably different. The algorithm reconstructs pure component spectra without relying on prior knowledge of the component spectra and the concentration profiles. This avoids many difficulties encountered with rule-based approaches or "traditional" background subtraction routines. Recently, we re-examined the possibility of applying BTEM to mass spectral data to recover pure component spectra. The primary difficulties include the discrete nature of mass spectra and the fact that fragmentation patterns can be rather similar due to the presence of many common fragments or adducts. Modifications of the original BTEM algorithm have now been performed to take into consideration the above mentioned characteristics of MS. In particular, the objective function was reformulated in terms of intensity (rather than derivatives), and a two-peak/band targeting strategy was employed to perform the spectral reconstruction. We call this modified algorithm tBTEM, emphasising the two-peak/band targeting concept, instead of single peak targeting concept in BTEM.

At this point, a brief mention of our interest in extending BTEM to discrete MS data is perhaps very useful in clarifying questions concerning applicable types of physical problems, and why reference spectra or expert knowledge are of little or no help. Here we can mention (1) exploratory studies of plasmas frequently used in chemical vapour deposition in the electronics/ semiconductor manufacturing industry for surface modification and the desire to understand the plasma chemistry and (2) exploratory studies of new and promising homogeneous metal mediated pharmaceutical syntheses and the desire to understand the transient intermediates present. In the first, on-line time-of-flight MS is a potential tool; in the second, on-line MS electrospray may be applicable. Since both types of systems can be anticipated to possess dozens of new, previously unobserved chemical entities, the analytical problems are difficult at best, and require the development of a new general approach. The primary goal of this contribution is to report the successful application of tBTEM to smaller test problems.

\section{Computational Details of tBTEM}

The BTEM algorithm has been successfully applied to many real systems [14-19] and has shown its considerable usefulness in finding unknown components and dealing with the pure component spectra of unstable species [16]. However, these applications involved only continuous spectra like FTIR and Raman. Difficulties were encountered when the BTEM algorithm was initially applied without modification to discrete spectra, e.g., mass spectral measurements. Thus modifications to the original BTEM algorithm were necessary so that mass spectra can be reconstructed. These modifications included: (1) Reformulating the objective function using the peak heights instead of their derivatives; (2) weighting the abstract vectors $V^{T}$ to reduce the effect of noise; (3) using a two-peak targeting strategy (tBTEM) to deal with strongly overlapping peaks.

\section{Overall tBTEM Algorithm}

The salient features of the tBTEM algorithm can be expressed in the following Steps.

1. Perform singular value decomposition (SVD) of spectroscopic data array $A_{k \times v}$ where $k$ is the number of experiments taken, $v$ is the number of spectroscopic data channels $(k<v)$. After truncating the physical meaningless parts of right singular matrix $V^{T}{ }_{v \times v}$ and zero part of diagonal matrix $\Sigma_{k \times v}$, they reduced to $V_{k \times v}^{T}$ and $\Sigma_{k \times k}$ [22-24].

$$
A_{k \times v}=U_{k \times k} \times \Sigma_{k \times v} \times V_{v \times v}^{T}
$$

2. Inspect the right-singular vectors in matrix $V^{T}{ }_{k \times v}$ to identify last $k-j$ vectors that appear to represent only noise. Discard these $k-j$ vectors. This leaves the 
truncated right singular matrix $V_{j \times v}^{T}$; i.e., the number of $V^{T}$ vectors which will be further used.

3. Identify an interesting local extrema in the first $j$ singular vectors to be targeted by tBTEM. Usually, only the extrema positions will be chosen in discrete spectra.

4. A set of random numbers is used as an initial guess for the first test vector $T_{1 \times j}$. $T$ is later updated by an appropriate global optimization method. The estimated spectrum $a^{\text {est }}$ is given by eq 2 where $S_{\mathrm{j} \times \mathrm{j}}$ is the weighting obtained from the singular values.

$a_{1 \times v}^{e s t}=T_{1 \times j} \times\left(S_{j \times j} \times V_{j \times v}^{T}\right)$

5. Normalize the estimated spectrum $\boldsymbol{a}^{\text {est }}$ by the maximum peak. Let the normalized spectrum be denoted as $\hat{a}$. Two peaks $a^{\prime}$ and $a^{\prime \prime}$ are usually chosen as targeted peaks in tBTEM.

$\hat{a}_{1 \times v}=\frac{a_{1 \times v^{e s t}}}{a^{\prime}+a^{\prime \prime}}$

6. Formulate the objective function (eq 4) in terms of the normalized $\hat{a}$

$$
\begin{aligned}
& \min (G)=\sum_{v} \hat{a}_{1 \times v}+P\left(\hat{\boldsymbol{a}}_{1 \times \boldsymbol{v}}, \hat{\boldsymbol{c}}_{k \times 1}\right) \\
& P\left(\hat{\boldsymbol{a}}_{1 \times \boldsymbol{v}}, \hat{\boldsymbol{c}}_{k \times 1}\right)=\gamma_{a} F_{a}\left(\hat{\boldsymbol{a}}_{1 \times \boldsymbol{v}}\right)+\gamma_{c} F_{c}\left(\hat{\boldsymbol{c}}_{k \times 1}\right) \\
& F_{a}\left(\hat{\boldsymbol{a}}_{1 \times \boldsymbol{v}}\right)=\sum_{v}\left(\hat{\boldsymbol{a}}_{\boldsymbol{v}}\right)^{2} \forall \hat{\boldsymbol{a}}_{\boldsymbol{v}} ;<0 \\
& F_{c}\left(\hat{\boldsymbol{c}}_{k \times 1}\right)=\sum_{k}\left(\hat{\boldsymbol{c}}_{k}\right)^{2} \forall \hat{\boldsymbol{c}}_{k} ;<0 \\
& \boldsymbol{\gamma}_{a}=\mathbf{1 0}^{\mathbf{4}} ; \boldsymbol{\gamma}_{b}=\mathbf{1 0}^{\mathbf{3}} ;
\end{aligned}
$$

The penalty function $P$ defined in eq 5 is used to guarantee non-negativity in reconstructed spectrum and concentrations. The estimated concentrations are defined in eq 7 .

$$
\hat{\boldsymbol{c}}_{k \times 1}=A_{k \times v} \times \hat{\boldsymbol{a}}_{\boldsymbol{v} \times 1}^{T} \times\left(\hat{\boldsymbol{a}}_{\boldsymbol{v} \times 1} \times \hat{\boldsymbol{a}}_{\boldsymbol{v} \times 1}^{T}\right)^{-1}
$$

7. Check the objective function value against a stopping criterion. If the stopping criterion is not met then generate another $\boldsymbol{T}$. Then repeat Steps 4 to 7 until one pure component spectrum is reconstructed.

8. Repeat Steps 3 to 8 by targeting other extrema to find the remaining pure component spectra.

\section{Utility of tBTEM and Crucial Aspects} of the Algorithm

The utility of tBTEM in solving the "blind" spectral reconstruction problem (e.g., given no prior information) arises because (1) in most practical situations, no assumption concerning the number of observable species is necessary, (2) spectral quality after reconstruction is greatly improved because non-linearities can be taken into account, and (3) the algorithm is goal orientedone targets an observed feature in $V^{T}$ and then recovers the associated entire function (pure component spectrum).

The right singular matrix $\boldsymbol{V}^{\boldsymbol{T}}$ reveals a crucial initial untangling of the pure component spectra. In tBTEM, a large number of vectors $j \gg s$ are taken from $V^{T}$ and these are then transformed, one-spectrum-at-a-time, into the pure component spectral estimates. The actual spectral recovery in tBTEM algorithm is initiated by targeting a feature in the decomposition $V^{T}=\left[V_{\nu \times k}\right.$ $\left.V \nu_{\nu \times(\nu-k)}\right]^{T}$, where only the first $k$ vectors have physical meaning ( $k$ measurements were made). The algorithm retains this interesting spectral feature and forces a reconstruction of the associated entire pure component spectrum.

Spectral simplicity and entropy. In BTEM for FTIR, Raman, and NMR spectra, entropy is evaluated in terms of spectral derivatives. However, in order to develop tBTEM for discrete MS data, the ability to differentiate was lost. As a consequence, the algorithm resorts to a minimization over the summation of the channel intensities. Although such a summation is rather removed from some of the original concepts of entropy, its minimization is nevertheless consistent with the spirit behind the principle of simplicity [9]. Due to this reasoning as well as the similarity of the algorithms, the root acronym has been retained. Finally, and in the interest of completeness, it should be noted that although a logarithm is often used in formulations of entropy, its omission allows the algorithm to run somewhat faster. Non-logarithmic forms of entropy exist in the literature $[25,26]$.

Weighting. In the original BTEM algorithm, since nonlinearities are present, a very large number of vectors are chosen, more than the number of species anticipated in the system. However, if far too many vectors are chosen, and hence far too much noise is introduced, problems can be encountered, particularly during the optimization procedure.

Because the vectors associated with both real signals and noise play equal roles in the original BTEM algorithm, adverse effects on the spectral reconstruction may be introduced particularly for discrete MS spectra. Obviously, it would be advantageous to lower the affects of noise. Therefore, in tBTEM for MS data, instead of using the $V^{T}$ vectors directly, we multiply the $V^{T}$ matrix with a set of weights (eq 2), namely the 
diagonal matrix $S$ that is readily available from SVD as mentioned in the section Computational Details of tBTEM, subsection Overall tBTEM Algorithm, Step 1. By scaling the right singular vectors, the importance of the noisy vectors are reduced during the optimization. Accordingly, it is less likely that the optimization method becomes trapped in a local minimum. In other words, the optimization is less sensitive to the choice of the number of $V^{T}$ vectors.

Two-peak/band targeting. In Step 5 of the tBTEM algorithm, (see section cited above) the estimated spectrum was normalized using the maximum value of the targeted band(s). Since the targeted peak plays a central role in retrieving the entire spectrum, the use of a higher peak is advantageous because of the larger signal to noise ratio and the resulting spectral estimate is often less affected by the noise present. For systems having moderately overlapping spectra, the BTEM algorithm is usually quite successful in estimating all the pure spectra using their highest peaks as targets. However, if strongly overlapping spectra are present at the highest peak position/channel, only the spectrum with smallest objective function value can be recovered with the original BTEM. To deal with this challenge, a two-peak (band) targeting strategy in tBTEM is used. (Note that tBTEM reduces to BTEM when $\mathrm{a}^{\prime}$ and $\mathrm{a}^{\prime \prime}$ are the same).

The best spectral reconstructions with tBTEM are obtained when the two highest peaks of a spectrum are used/targeted. Since the chances are small that two pure component spectra have their highest peaks located at the same positions/channels, permutations for different pairs of highest peaks over the entire spectral range provides an exhaustive search for pure component spectra. Consequently, it is possible to recover most if not all the pure component spectra. If extremely overlapping pure component spectra are suspected in the system, e.g., more than two highest peaks overlap or one spectrum is almost totally overlapping with another spectrum, a multiple targeted peak strategy might be employed to handle such a complex system eq 8 .

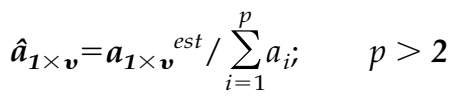

\section{Simulation Results}

In this section, the use of the tBTEM algorithm is examined, and the consequences for sets of mass spectra with strongly overlapping spectroscopic features are noted. Experimental MS pure component spectra were measured and synthetic mixture data were generated from these experimental spectra. The sampling frequency is low and minor white noise is added to the spectra. The low level noise is not so important for the conclusions of this section since the immediate goal is to examine the intrinsic capability of the new algorithm.

A system with ten experimental pure components
Table 1. The ten components in the simulated system

\begin{tabular}{lcc}
\hline Component & $\begin{array}{c}\text { Molecular } \\
\text { weight }\end{array}$ & $\begin{array}{c}\text { Highest peak } \\
\text { position }(\mathrm{m} / \mathrm{z})\end{array}$ \\
\hline \hline Ethanol & 45 & 31 \\
Hexane & 86 & 57 \\
Toluene & 92 & 91 \\
Acetone & 58 & 43 \\
Acetonitrile & 41 & 41 \\
Cyclohexene & 84 & 56 \\
$\left(\mathrm{CH}_{3}\right)_{2} \mathrm{CHOH}$ & 60 & 45 \\
$\mathrm{Acetic}$ acid & 60 & 43 \\
$\mathrm{CH}_{2} \mathrm{Cl}_{2}$ & 84 & 49 \\
$\mathrm{CH}_{3} \mathrm{CH}_{2} \mathrm{COCH}$ & 72 & 43 \\
\hline
\end{tabular}

was considered (Table 1). The MS spectra of the ten reference components were measured with a range of $m / z=10-100$ with a resolution $1 \mathrm{~m} / \mathrm{z}$. The ten spectra were obtained from GC-MS (GC: Hewlett-Packard 6890, MS: Hewlett-Packard 5973) with the EI ionization method and helium as mobile phase.

Fifty mixtures containing the ten components were simulated with randomly generated non-negative concentrations for each mixture (arbitrary units, range from 0 to 100). Accordingly, a concentration matrix for the 50 mixtures $C_{50 \times 10}$ was generated. For convenience, all pure component spectra were first scaled with a maximum peak height of $10^{6}$ (arbitrary unit). The mass spectra of the mixtures were simulated using eq 9 where $\boldsymbol{P}$ denotes the pure spectra of the ten components and $\varepsilon$ the random noise matrix with elements 0 to $10^{4}$. In the present study, the total channels for each spectrum is 91 .

$$
A_{50 \times 91}=C_{50 \times 10} \times P_{10 \times 91}+\varepsilon_{50 \times 91}
$$

As anticipated, the simulated mixture spectra are very complex. Since many of the components have common functional groups such as $\mathrm{CH}_{3}-, \mathrm{CH}_{3} \mathrm{CH}_{2}-, \mathrm{CH}_{3} \mathrm{CO}-$ etc. many common fragments occur, namely $\mathrm{CH}_{3}^{-}(\mathrm{m} / \mathrm{z}$ 15), $\mathrm{CH}_{3} \mathrm{CH}_{2}\left(\mathrm{~m} / z\right.$ 29), and $\mathrm{CH}_{3} \mathrm{CO}^{\circ}(\mathrm{m} / z$ 43). Consequently the MS spectra of many components are highly overlapping.

The simulation and the spectral reconstruction using tBTEM were developed on the commercial software MATLAB 5.3 and run on a Toshiba laptop with Intel Pentium III $800 \mathrm{MHz}$ and $384 \mathrm{M}$ Ram. The computation times taken for every pure spectral reconstruction varied from 3 to $30 \mathrm{~min}$, depending on the number of $V^{T}$ used for a reconstruction. The optimization algorithm employed in tBTEM was Corana's simulated annealing [27].

\section{Performance of Modified Objective Function for Discrete Spectra}

The performance of the modified objective function (eq 4-6) was tested. The tests were performed with the simulated mass spectral data by using the first $10 \mathrm{~V}^{T}$ vectors and targeting only one peak in these initial tests. 
Table 2. Result of estimated spectra compared with real spectra

\begin{tabular}{|c|c|c|c|c|}
\hline \multirow[b]{2}{*}{ Component } & \multirow{2}{*}{$\begin{array}{c}\text { Real spectra } \\
\text { objective function } \\
\text { value }\end{array}$} & \multicolumn{3}{|c|}{ Estimated spectra } \\
\hline & & $\begin{array}{c}\text { Peak targeted } \\
\text { at }(\mathrm{m} / \mathrm{z})\end{array}$ & $\begin{array}{c}\text { Objective } \\
\text { function value }\end{array}$ & $\begin{array}{c}\text { Inner } \\
\text { production }\end{array}$ \\
\hline Ethanol & 4.7648 & 31 & 4.7601 & 1.00000 \\
\hline Hexane & 6.6033 & 57 & 6.5028 & 0.99983 \\
\hline Toluene & 4.4888 & 91 & 4.4761 & 0.99996 \\
\hline Acetone & 4.7731 & 58 & 4.645 & 0.99737 \\
\hline Acetonitrile & 2.9309 & 41 & 2.9305 & 1.00000 \\
\hline Cyclohexene & 3.9546 & 56 & 3.8604 & 0.99776 \\
\hline$\left(\mathrm{CH}_{3}\right)_{2} \mathrm{CHOH}$ & 2.9658 & 45 & 2.8805 & 0.99886 \\
\hline Acetic acid & 4.7739 & 60 & 4.7714 & 0.99996 \\
\hline $\mathrm{CH}_{2} \mathrm{Cl}_{2}$ & 3.8334 & 49 & 3.8312 & 1.00000 \\
\hline $\mathrm{CH}_{3} \mathrm{CH}_{2} \mathrm{COCH}_{3}$ & 2.9028 & 43 & 2.8536 & 0.99972 \\
\hline
\end{tabular}

Note that the number of $V^{T}$ vectors used is equal to the number of the pure components in the system. The result is shown in Table 2 where the targeted peak and the value of the objective function are given. For comparison purpose, the objective function values of all the real pure spectra are also calculated and listed. It is evident that both the measured and reconstructed objective function values are very similar, where the latter is usually slightly smaller.

The quality of the reconstructions was further examined by taking the inner product (IP) of the estimated and corresponding real spectra. Although more sophisticated measures of comparison exist [28], the inner product provides a very simple measure of the degree of similarity between two spectra. When the two compared spectra are normalized to unit vectors, the IP value should fall in the range of 0 to 1 . If two spectra match exactly, the IP equals 1 . On the other hand, if the IP approaches 0, the two spectra are orthogonal. Obviously, all the IP's are very close to 1 which is further confirmation of the excellent reconstructions of all the component spectra.

\section{Comparison of Weighted and Unweighted $V^{T}$ Vectors}

On the basis of the aforementioned results, the number of $V^{T}$ vectors used was increased to 50 . This is the same as the number of mixtures synthesized. No weights are introduced. It was found that the reconstructed pure component spectra are in general not very acceptable. For example, the estimated cyclohexane spectrum (Figure 1c) does not resemble the real one (Figure 1a). The result was improved greatly when the $50 V^{T}$ vectors were weighted by the diagonal matrix $S$ according to eq 2 (results shown in Figure 1b). This indicates that the weighting makes the algorithm less sensitive to the choice of the number of used $V^{T}$ vectors. Indeed, although a lot of noise is present in the $50 V^{T}$ vectors used, the performance of the algorithm is very good. It is important to stress that this weighting procedure is very helpful in spectral reconstruction from real exper- imental systems where the exact number of components in the system is unknown.

Inspection of Figure 1 clearly indicates that objective function value of the spectrum in Figure 1c is larger than that of the spectrum in Figure 1b. This type of result occurs often when many $V^{T}$ vectors are used. The un-weighted algorithm produces an incorrect pure component spectrum while the weighted algorithm produced an excellent reconstruction. The weighting/ scaling of $V^{T}$ improves the results considerably.
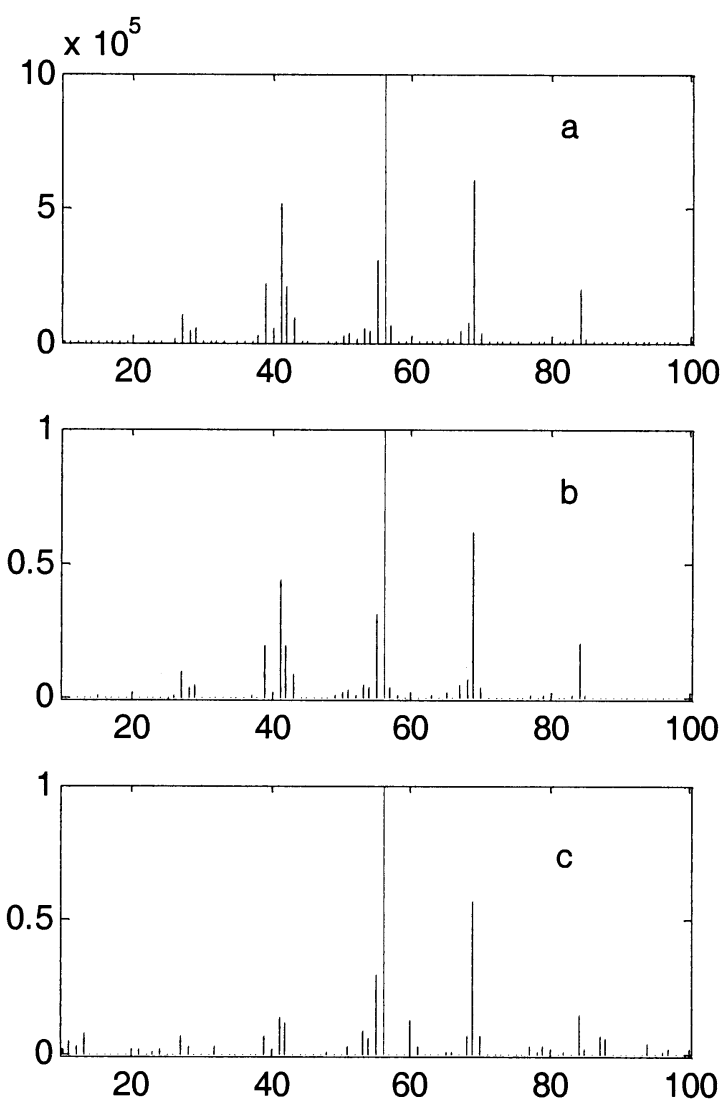

Figure 1. The real experimental and estimated cyclohexane spectra. (a) Real experimental spectrum; (b) estimated spectrum using 50 weighted $V^{T} ;\left(\right.$ c) estimated spectrum using 50 unweighted $V^{T}$. 
Table 3. Comparison of objective function values by using different targeted peaks

\begin{tabular}{lccc}
\hline Target at $(\mathrm{m} / \mathrm{z})$ & $\mathrm{CH}_{3} \mathrm{CH}_{2} \mathrm{COCH}_{3}$ & $\mathrm{CH}_{3} \mathrm{COOH}_{3}$ & $\mathrm{CH}_{3} \mathrm{COOH}$ \\
\hline \hline 43 only & 2.9028 & 3.5342 & 3.5388 \\
43 and 58 & 2.8837 & 2.0306 & 3.5388 \\
43 and 60 & 2.9025 & 3.5156 & 2.0323
\end{tabular}

\section{Comparison of tBTEM and BTEM}

As was shown in Table 2, the single peak targeting algorithm was able to identify the pure component spectra for $\mathrm{CH}_{3} \mathrm{CH}_{2} \mathrm{COCH}_{3}, \mathrm{CH}_{3} \mathrm{COCH}_{3}$, and $\mathrm{CH}_{3} \mathrm{COOH}$. Note that the targeted peak for the reconstruction of $\mathrm{CH}_{3} \mathrm{CH}_{2} \mathrm{COCH}_{3}$ was $\mathrm{m} / \mathrm{z} 43$, the highest peak, while the reconstructions of $\mathrm{CH}_{3} \mathrm{COCH}_{3}$ and $\mathrm{CH}_{3} \mathrm{COOH}$ used $\mathrm{m} / \mathrm{z} 58$ and 60 which are not the highest peaks for these two spectra.

Difficulties may be encountered, particularly with BTEM, if the highest peaks of two or more spectra are all located at the same channel $(\mathrm{m} / \mathrm{z})$. This is because both the global and some of the local minima (obtained during the optimization) correspond to the real pure component spectra. In BTEM, only the global minimum is kept and the local minima are discarded. As a result, only one spectrum will be recovered. For example, in the present data set, $\mathrm{CH}_{3} \mathrm{CH}_{2} \mathrm{COCH}_{3}, \mathrm{CH}_{3} \mathrm{COCH}_{3}$ (acetone), and $\mathrm{CH}_{3} \mathrm{COOH}$ (acetic acid) have their respective highest peaks located at $\mathrm{m} / \mathrm{z} 43$-indicative of the fragment $\mathrm{CH}_{3} \mathrm{CO}^{\circ}$. When the peak at $m / z \quad 43$ was chosen, the algorithm was successful in reconstructing the spectrum of $\mathrm{CH}_{3} \mathrm{CH}_{2} \mathrm{COCH}_{3}$ with the minimum objective function value of 2.9028. In contrast, although the local minimum objective function values of 3.5342 and 3.5388 corresponded to the spectral solutions of $\mathrm{CH}_{3} \mathrm{COCH}_{3}$ and $\mathrm{CH}_{3} \mathrm{COOH}$, they were discarded during global search. Therefore, BTEM, using only a single targeted peak could not find the spectra of $\mathrm{CH}_{3} \mathrm{COCH}_{3}$ and $\mathrm{CH}_{3} \mathrm{COOH}$ using the highest peak $m / z=43$.

The tBTEM algorithm has the ability to circumvent this difficulty. By choosing two big peaks (usually the highest two peaks) of a spectrum, tBTEM obtains the minimum objective function corresponding to the solution of one of the spectra. Then in another run, another pair of highest peaks is used and a new solution corresponding to another spectrum is obtained. For example, in Table 3 the use of the two highest peaks $\mathrm{m} / \mathrm{z}$ equal 43 and 58 resulted in the minimum objective function 2.0306 and the spectral reconstruction $\mathrm{CH}_{3} \mathrm{COCH}_{3}$. Similarly, the choice of the two highest peaks of $\mathrm{m} / \mathrm{z}$ equal 43 and 60 resulted in an objective function 2.0323 and spectral reconstruction of $\mathrm{CH}_{3} \mathrm{COOH}$.

Moreover, in comparison with BTEM algorithm, tBTEM is less sensitive to the number of $V^{T}$ vectors used. For example, when $35 V^{T}$ vectors were used, the resultant spectra of hexane obtained from the BTEM (targeted at $m / z=57$ ) and $\mathrm{tBTEM}$ (targeted at $\mathrm{m} / \mathrm{z} 57$ and 86 ) were obtained (Figure $2 c$ and Figure $2 b$, respec-
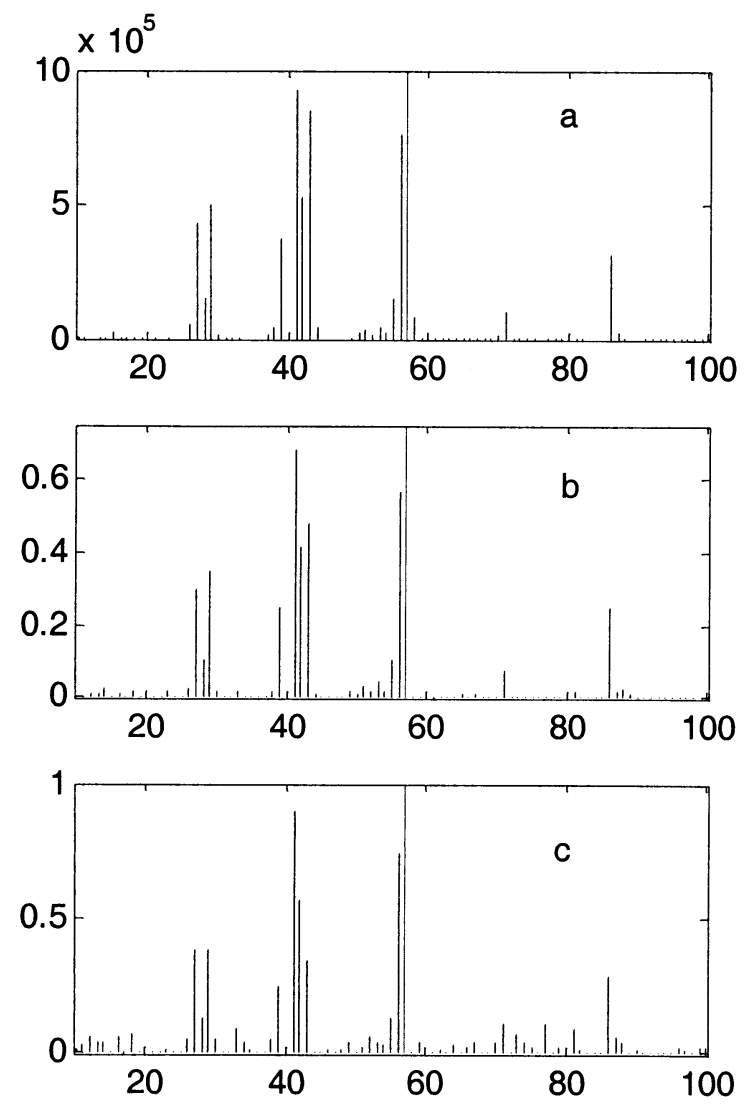

Figure 2. Hexane spectra by using one peak or two peaks for targeting (35 $\mathbf{V}^{\mathrm{T}}$ used). (a) Real experimental spectrum; (b) estimated spectrum resulting from targets at $\mathrm{M} / \mathrm{e} 57$ and $\mathrm{M} / \mathrm{e} 86$; (c) estimated spectrum resulting from target at $\mathrm{M} / \mathrm{e} 57$ only.

tively). Clearly, the resulting spectrum from tBTEM resembles the real one very closely (Figure 2a) while a higher level of noise is present in the spectral reconstruction from BTEM. The inner products of real and the estimated spectra from tBTEM and BTEM are 0.99366 and 0.95635 , respectively. This again shows that tBTEM outperforms the single targeted peak algorithm.

The improvement in the performance of tBTEM appears because of the use of more spectral information, i.e., two peaks instead of only a single peak. The enriched spectral information facilitates the reconstruction of pure component spectra from highly overlapped mixture spectra.

\section{Exhaustive Search}

For either the BTEM or the tBTEM algorithms, each pure spectrum is recovered from a separate run. Therefore, many runs are needed in order to recover all pure spectra, and these runs are directed by the user's choice of the targeted peaks. Clearly, a problem arises when many channels of data must be considered. Recovery of all potential pure component spectra may not be realistic/practical if the search is left to the user's judgement alone. Accordingly, an automated exhaustive search is desirable. 
The exhaustive search starts with the full permutation of the targeted peaks to generate a super-set of estimated spectra. It is clear that such a super-set will contain not only real pure component spectra but also occasionally some not-so-real spectra which arise from the superposition (additivity) of pure component spectra (the latter often arise when two smaller peaks are targeted). Obviously, a few heuristic rules are useful in eliminating non-real spectra and eventually identify the real component spectra. In order to describe the exhaustive search, we will use the simulated data with tBTEM as discussed in the previous sections.

\section{Full Permutation of Targeted Peak Pairs}

Let $\mathrm{m}$ denote the number of channels in the spectroscopic data. Then a full permutation of any pair of peaks (channels), from the entire set of channels leads to $\mathrm{n} \gg \mathrm{m}$ combinations. In other words, $\mathrm{n}$ runs are required and $n$ estimated spectra are obtained.

In most real physical problems, a full permutation over the set of $\mathrm{m}$ channels is not necessary. Indeed, many channels will have little or no real physical information. Accordingly, let $\mathrm{m}^{\prime}$ denote the number of channels considered after the data (the first few $V^{T}$ vectors) are filtered. This filtering can be automated, thereby eliminating channels having less than a critical threshold value, or performed manually, whereby the user inspects the data set and identifies a sub-set of interest. The latter may be more efficient (since a smaller set $\mathrm{m}^{\prime}$ is identified) but it may require inclusion of experience/some expert knowledge. Then, the realistic number of runs $n^{\prime}$ required, and $n^{\prime}$ estimated spectra generated are:

$$
n^{\prime}=C_{2}^{m}=m^{\prime} \times\left(m^{\prime}-1\right) / 2
$$

Following the idea further, in order to develop the automated routine, the targeted peaks are chosen from the first $V^{T}$ vector using the threshold method. The set of targeted peaks chosen in this way seems reasonable as the first $V^{T}$ vector represents an average of total mixture spectrum. A rather stringent threshold can be set if desired, thus maximizing the admissible channels to be searched. A useful threshold can be easily defined as the ratio of a peak to the maximum value in the first $V^{T}$. For the simulated system considered here, if the threshold is set to 0.05 (Case 1), then $m^{\prime}$ is equal to 33; while if the threshold is 0.1 (Case 2) then $m^{\prime}$ becomes 26 . As a result, the total number of the estimated spectra $n^{\prime}$ for Cases 1 and 2 are 528 and 325, respectively.

\section{Initial Filtering/Rejection of Undesirable Estimated Spectra}

A real spectrum can always be reconstructed by tBTEM using an exhaustive search. Also, the reconstruction is expected to possess a high degree of accuracy if the highest peak is targeted because the highest peak has larger signal to noise ratio. The first rule therefore rejects those estimated spectra whose highest peak is not one of the two targeted peaks. The collection of the candidate spectra is thus reduced. For Cases 1 and 2, the number of the candidates are reduced to 67 and 58, respectively.

\section{Reject the Duplicate Spectra for a Real Component}

For real pure component spectra having a number of fragments, an exhaustive search using tBTEM will always result in multiple estimates of the real spectrum. In order to detect and reject these duplicate estimates, the inner product of every two estimated spectra can be calculated after normalizing to unit vectors. Due to the presence of noise, a threshold of the Inner Product can be set, e.g., a typical value may be 0.95 . If the resulting inner product is larger than 0.95 , one of the two spectra under investigation is regarded as the duplicate and is rejected from the collection of potential candidates. The spectral estimate with the smaller objective function is kept while the other is discarded. In contrast, if the inner product is less than 0.95 , both spectra are regarded as promising and thus kept for further examination. In doing so, the numbers of the candidate spectra in the simulated system decrease to 24 and 22 for Cases 1 and 2, respectively.

\section{Reject the Spectra That Have Linear Relationships with Other Spectra}

The third rule rejects spectral estimates which are linear combinations of other spectra. Assume that a spectrum $\boldsymbol{X}$ is linearly combined by two other spectra $\boldsymbol{Y}$ and $\boldsymbol{Z}$, then the following relationship holds, where $\boldsymbol{\alpha}$ and $\boldsymbol{\beta}$ are positive coefficients, and err represents the vector of errors which should approach zero. A full permutation of combinations for $\boldsymbol{X}, \boldsymbol{Y}, \boldsymbol{Z}$ are searched to reveal linear dependent combinations, within some prespecified tolerance for the term $e r r$.

$$
X=\alpha \times Y+\beta \times Z+e r r
$$

In the present implementation of this rule, a variety of methods were used in order to identify these independent component spectra. These include the use of least squares to obtain coefficients and comparison of spectra (vectors) using inner products. The latter requires a user defined tolerance.

During application of the rule, spectral estimates that are linear combinations (in the sense of eq 11 with positive coefficients) are removed from the collection of the candidate component spectra. After checking the full permutation of estimated spectra $X, Y$, and $Z$, the set of remaining spectra is reduced in size, and these are regarded as the pure component spectral estimates for the system. For the present simulated system, the final 
numbers of the remaining spectra for both Case 1 ( $\mathrm{n}^{\prime}=$ $528)$ and Case $2\left(n^{\prime}=325\right)$ are reduced exactly to 10 using reasonable values for the tolerance, namely 0.98 for inner product. These 10 resultant estimates did in fact correspond to the 10 reference component used in the simulation.

\section{Computational Considerations}

The spectral reconstruction algorithm tBTEM has to be executed hundreds of times during the exhaustive targeting. This represents the primary computational demand in the exhaustive search. Indeed, a typical run time for each run of tBTEM was on the order of a few minutes, and therefore, the few hundred executions required for Case 1 and Case 2 required on the order of $10 \mathrm{~h}$. These calculations were performed on a 2GB RAM, Intel Xeon $500 \mathrm{MHz}$, Windows NT workstation. After the generation of the superset of spectral estimates, the heuristic rules are applied. This latter sorting and reduction of the set of spectral estimates requires little time compared to the numerous global searches required for the simulated annealing driven tBTEM.

\section{Experimental Results}

In this section, the tBTEM algorithm is applied to an entirely experimental set of mass spectra arising from real mixtures of four components, namely ethanol, acetone, hexane, and toluene. The mixture mass spectra were obtained from GC-MS equipped with a short capillary column which allowed only a small degree of separation and therefore only a small degree of variation in signal as a function of time.

\section{Challenges in the Real MS System}

There are differences between synthetic mixtures of reference spectra used in the section Simulation Results and real mixture mass spectra analyzed in this section (the EI method is used in both Cases). One difference is that fragments from the various components injected as a mixture may undergo some recombination and new signals may appear. The resulting spectral reconstructions from the major components may possess some contributions/omissions from these new signals, and these may even exist in channels where zero intensity is expected. This can lead to considerable complications when further identification is required.

Another still important difference is that the "pure" MS of a species may differ depending on the system background pressure etc. This latter condition is often referred to as a non-stationary signal in the signal processing literature. Although BTEM is known to be able to deal rather well with non-stationary signals from FTIR and Raman data, such non-linearities can be tolerated because of the relatively good $\mathrm{S} / \mathrm{N}$ ratio typical of these spectroscopies. Therefore, in MS data where $\mathrm{S} / \mathrm{N}$ ratios are typically lower, non-stationary pure component spectra may be more difficult to recover.

\section{Experimental Setup and Data Collection}

A GC-MS spectrometer (GC: Hewlett-Packard 6890, MS: Hewlett-Packard 5973) with a 5 m 100\% methyl stationary phase capillary column were used together with a helium mobile phase and a $1 \mu \mathrm{L}$ syringe. It should be noted that this column has low separation ability and therefore is usually used for retention gaps and guard columns in GC but not for separation purposes. The use of a $1 \mu \mathrm{L}$ syringe injection into the GC-MS system lowers the level of fragment recombination because of the lower overall amount of solutes/ solvent. Each mixture sample was injected slowly and smoothly, resulting in a somewhat flat GC peak at the detector. Consequently, little separation of components occurred.

Five samples were prepared from a random mixture of four low molecular weight compounds, i.e., ethanol, hexane, toluene, and acetone. Each $1 \mu \mathrm{L}$ mixture sample was injected over a period of a few seconds. Some variation in the composition of the components occurred at the detector during the detection period. The range of the exported data was from $\mathrm{m} / \mathrm{z} 10$ to 100 with the interval of $1 \mathrm{~m} / \mathrm{z}$. As a result, a series of circa $400 \mathrm{MS}$ spectra data were acquired from the five sample injections. Only a small number of these spectra were used in the analysis. The manually selected mass spectra were collected in the more-or-less flat regions of the total ion count (TIC). This yielded 16 mixture mass spectra used in the subsequent reconstructions.

\section{Results of the Spectral Reconstruction}

In the spectral reconstructions performed with $\mathrm{tBTEM}$, all $16 V^{T}$ vectors were weighed by the singular value matrix, i.e., the diagonal matrix $S$ of SVD. Each pure component spectrum was reconstructed by targeting two specified peaks: Ethanol 31 and 45, acetone 43 and 58 , hexane 57 and 86 , toluene 91 and 92 . The four pure component spectra were successfully recovered. They are compared to the experimental reference as shown in Figure 3.

The characteristic fragmentation patterns for the ethanol, acetone, hexane, and toluene are readily apparent in the spectral reconstructions-they are rather good. Closer comparison of these reconstructions with the "experimental references" indicates an interesting artefact. The reconstructions are in some ways "cleaner". Although the primary fragments are still very prominent, the intensities of some of the other fragments are reduced. This is the Case for all four component spectral reconstructions. This reduction in the intensity of the other channels is probably related to (1) the non-stationary quality of the pure mass spectra as well as (2) the role of entropy minimization to 

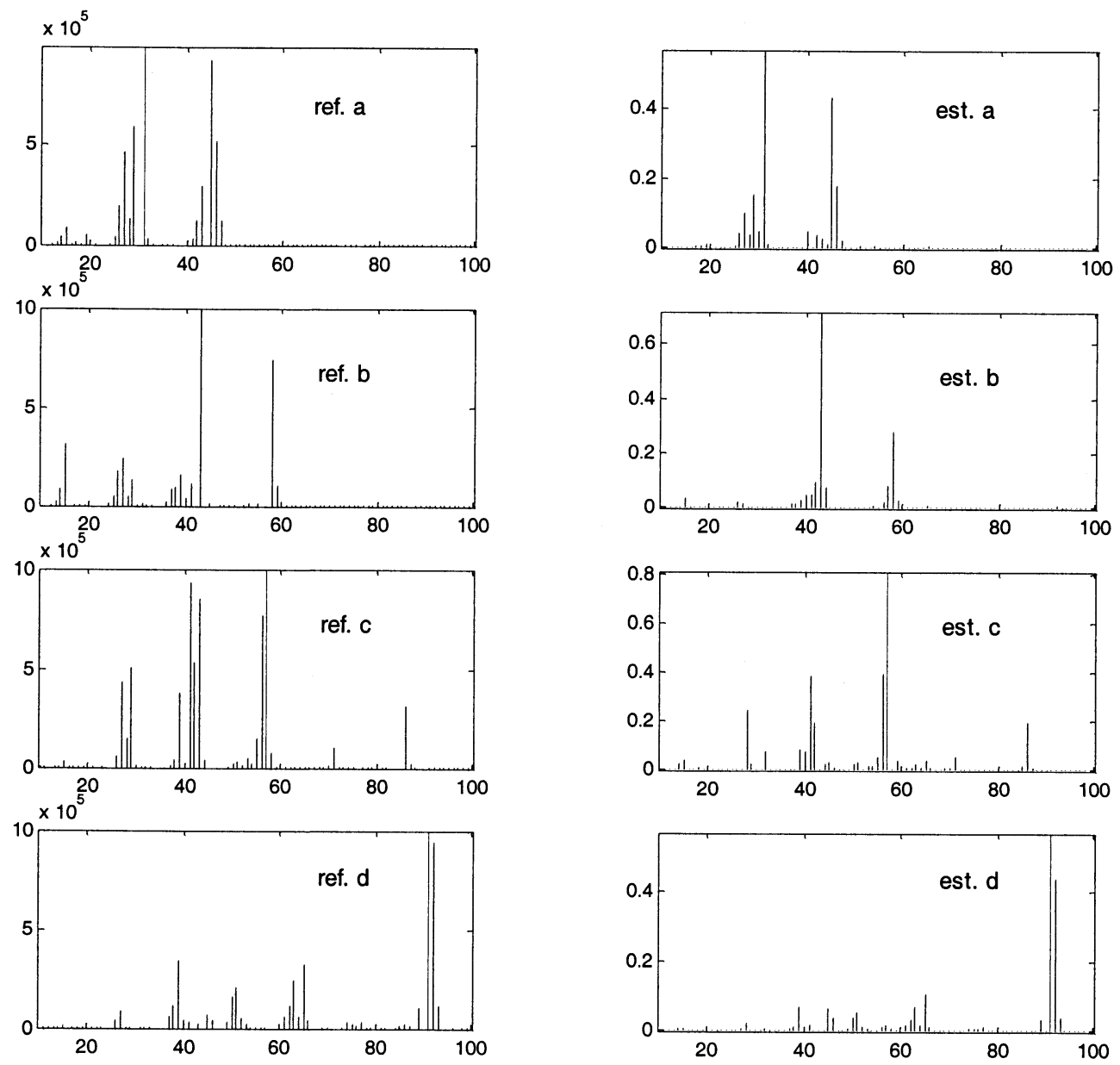

Figure 3. Comparison of experimental "reference" spectra with estimated pure spectra. Left column: References; right column: Estimated spectra. (a) Ethanol, (b) acetone, (c) hexane, and (d) toluene.

simplify spectra thus subtracting the presence of a low background of recombination fragments.

Again, it is important to emphasize the utility of using weighted $\boldsymbol{V}^{T}$ vectors. Ideally, if the experimenter had prior knowledge of the system, the number of the $V^{T}$ vectors used would be equal to the number of pure components in the system in order to get the best results i.e., four in the present example. However, the emphasis of the present contribution is for exploratory studies of unknown systems, and therefore it is assumed that information on the number is unknown a priori. Consequently, more $V^{T}$ vectors are used than really needed, but more noise is introduced. Figure $4 \mathrm{a}$ and $\mathrm{b}$ are the reconstructed ethanol spectra using unweighted four and six $\boldsymbol{V}^{T}$ vectors, respectively. As can be seen from these two figures, spectral features arising from toluene at $\mathrm{m} / \mathrm{z}$ 91 and 92 appear. This is more prominent in the six $V^{T}$ (Figure $4 b$ ) Case than the four $\boldsymbol{V}^{T}$ Case (Figure 4a). Clearly, the weighted $16 V^{T}$ vector solution presented in Figure 3 is much better.
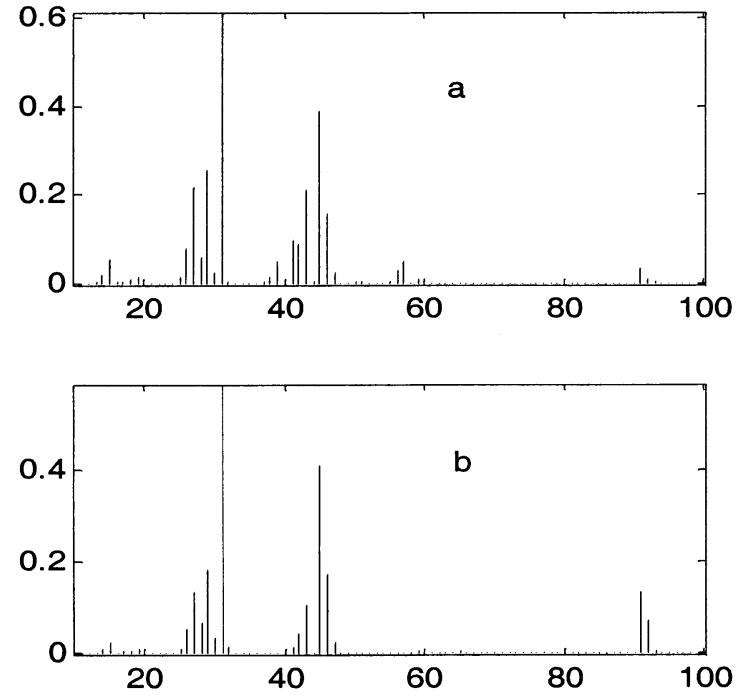

Figure 4. Results of estimated ethanol spectra using different number of $\mathbf{V}^{\mathrm{T}}$ vectors. (a) Using 4 unweighted $\mathbf{V}^{\mathrm{T}}$; (b) using 6 un-weighted $\mathbf{V}^{\mathrm{T}}$. 


\section{Discussions and Conclusions}

In this paper, modifications on the advanced spectral reconstruction algorithm-BTEM are proposed. These modifications extend the algorithm to handle discrete spectroscopic data like mass spectra and improve the performance of the algorithm.

By reformulating the objective function with the peak heights instead of their derivatives, the algorithm is able to reconstruct pure component spectra from mixture mass spectra. Furthermore, the algorithm is more computationally efficient as fewer mathematical operations are needed for the evaluation of the objective function.

Weighting of the abstract $V^{T}$ vectors reduces the adverse effect of noise and the sensitivity of choosing the number of $V^{T}$ vectors used. As a result, the algorithm is more robust. Weighting also allows a larger number of $V^{T}$ vectors to be used, thereby increasing the amount of recoverable information even in the presence of more noise.

A significant improvement in the reconstruction of highly overlapped spectra has been achieved by using the tBTEM algorithm where two peaks are used for targeting. In principle, the salient idea in tBTEM might be extended to deal with really highly overlapped spectra by considering multiple $(>2)$ targeted peaks.

The tBTEM algorithm is based on the idea that each component spectrum is reconstructed one-at-a-time based on the choice of chosen targeted peaks. Therefore, an exhaustive search provides a strategy for generating all possible pure component spectra present. The search executes tBTEM many times and then reduces the super-set of estimates to the sub-set of only real component spectra.

For real systems, difficulties can be encountered and these are related to the presence of non-stationary signals and the presence of fragment recombination. With the present experimental example, two difficulties arose, namely a slight simplification of the pure component spectra after tBTEM and difficulties with the exhaustive search. The difficulties observed here do not negate the possibility that analysis from other types of experimental configurations/methods may be easier. Good examples may include (1) ultra-high vacuum studies of desorbed species in surface science studies, (2) chemical vapor decomposition at low system pressures, (3) time-of-flight data from reactive systems, and (4) electrospray ionization mass spectrometry (ESI MS) from reactive systems. In the last Case the tBTEM may work rather well as fewer fragments are present and the spectral patterns are perhaps more stationary. A successful application to ESI MS may greatly facilitate spectral reconstruction in the fields of biochemistry and organometallics where complex and poorly separable mixtures are common.

Finally, as mentioned in the section Experimental Results, a poor performance GC column was used to induce a series of different experimental measurements from one sample for testing tBTEM. This idea might actually be of some utility when sample size is very small/limited. One does not have to find a really good column for the separation. Accordingly, one could use tBTEM plus a poor-performance GC column before MS to obtain pure component information. It would be possible to get good reconstructions from using any "poor" separation of sample on any arbitrarily "poor" column.

In summary, the performance of the tBTEM algorithm was examined with simulated and experimental mixture spectra. The recovered spectral estimates are quite acceptable. These tests suggest that tBTEM holds considerable potential for many real mass spectroscopy applications.

\section{Appendix}

\section{Nomenclature}

$A$, Experimental mixture data matrix

$\hat{a}$, Normalized estimated spectrum/spectra

$\hat{\boldsymbol{a}}^{\text {est }}$, Unnormalized estimated spectrum

$c$, Concentration of pure component (s)

$\hat{c}$, Estimated concentration

$F$, Penalty function term

$G$, Objective function value of BTEM or tBTEM

$h$, Shannon entropy measure

$P$, Penalty functions of BTEM and tBTEM

$\boldsymbol{P}$, Matrix contains pure spectra (10 reference spectra)

$S$, Square diagonal singular value matrix after truncation from $\Sigma$

$T$, Transform matrix/vector

$U$, Left singular matrix

$V^{T}$, Transposed right singular matrix

$X, Y, Z$, Estimated spectra which are linearly dependent

\section{Greek Letters}

$\Sigma$, Diagonal singular value matrix

$\alpha, \beta$, Linear coefficients of estimated spectra in exhaustive search

$\varepsilon$, Error matrix

$\gamma$, Coefficients of penalty function

\section{Subscripts}

a, Estimated spectrum/spectra

c, Estimated concentrations

$\mathrm{j}$, Number of right singular vectors used in optimization

$k$, Number of experiments

$\mathrm{s}$, Number of observable species in mixture

$v$, Number of channels of spectrum/spectra

\section{References}

1. Lawton, W. H.; Sylvestre, E. A. Interactive Self-Modeling Mixture Analysis. Technometrics 1971, 13, 617-633. 
2. Sylvestre, E. A.; Lawton, W. H. Maggio M. S. Curve Resolution Using a Postulated Chemical Reaction. Technometrics 1974, 16, 353-368.

3. Windig, W. Guilment J. Interactive Self-Modeling Mixture Analysis. Anal. Chem. 1991, 63, 1425-1432.

4. Windig, W. Spectral Data Files for Self-Modeling Curve Resolution with Examples Using the Simplisma Approach. Chemometr. Intell. Lab. Syst. 1997, 36, 3-16.

5. Bu, D. S.; Brown, C. W. Self-Modeling Mixture Analysis by Interactive Principal Component Analysis. Appl. Spectrosc. 2000, 54, 1214-1221.

6. Sanchez, F. C. Toft, J., Van den Bogaert B.; Massart, D. L. Orthogonal Projection Approach Applied to Peak Purity Assessment. Anal. Chem. 1996, 68, 79-85.

7. Sasaki, K.; Kawata, S.; Minami, S. Constrained Nonlinear Method for Estimating Component Spectra from Multicomponent Mixtures. Appl. Optics 1983, 22, 3599-3603.

8. Watanabe, S. Pattern Recognition as a Quest for Minimum Entropy. Pattern Recog. 1981, 13, 381-387.

9. Kanpur, J. N. Maximum-Entropy Models in Science and Engineering; Wiley Eastern Ltd: New Delhi, 1993, p 3.

10. Zeng, Y.; Garland, M. An Improved Algorithm for Estimating Pure Component Spectra in Exploratory Chemometric Studies Based on Entropy Minimization. Anal. Chim. Acta. 1998, 359, 303-310.

11. Pan, Y.; Susithra, L.; Garland, M. Pure Component Reconstructions Using Entropy Minimizations and VarianceWeighted Piecewise-Continuous Spectral Regions. Application to the Unstable Experimental System $\mathrm{Co}_{2}(\mathrm{CO})_{8} /$ $\mathrm{Co}_{4}(\mathrm{CO})_{12}$. J. Chemometrics 2000, 14, 63-77.

12. Widjaja, E.; Garland, M. Pure Component Spectral Reconstruction from Mixture Data Using SVD, Global Entropy Minimization, and Simulated Annealing. Numerical Investigations of Admissible Objective Functions Using a Synthetic 7-Species Data Set. J. Comput. Chem. 2002, 23, 911-919.

13. Chen, L.; Chew, W.; Garland, M. Spectral Pattern Recognition of in situ FTIR Spectroscopic Reaction Data Using Minimization of Entropy and Spectral Similarity (MESS). Application to the Homogeneous Rhodium Catalyzed Hydroformylation of Isoprene. Appl. Spectrosc. 2003, 57, 491-498.

14. Chew, W.; Widjaja, E.; Garland, M. Band-Target Entropy Minimization (BTEM): An Advanced Method for Recovering Unknown Pure Component Spectra. Application to the FTIR Spectra of Unstable Organometallic Mixtures. Organometallics 2002, 21, 1982-1990.

15. Widjaja, E.; Li, C.; Garland, M. Semi-Batch Homogeneous Catalytic In-Situ Spectroscopic Data. FTIR Spectral Recon- structions Using Band-Target Entropy Minimization (BTEM) without Spectral Preconditioning,. Organometallics 2002, 21, 1991-1997.

16. Li, C.; Widjaja, E.; Chew, W.; Garland, M. Rhodium Tetracarbonyl Hydride: The Elusive Metal Carbonyl Hydride. Angew. Chem. Int. Ed. 2002, 41(20), 3785-3789.

17. Li, C.; Widjaja, E.; Garland, M. The $\mathrm{Rh}_{4}(\mathrm{CO})_{12}$ Catalyzed Hydroformylation of 3,3-Dimethylbut-1-ene Promoted with $\mathrm{HMn}(\mathrm{CO})_{5}$. Bimetallic Catalytic Binuclear Elimination as an Origin for Synergism in Homogeneous Catalysis. J. Am. Chem. Soc. 2003, 125, 5540-5548.

18. Li, C.; Widjaja, E.; Garland, M. Spectral Reconstruction of In-Situ FTIR Spectroscopic Reaction Data Using BandTarget Entropy Minimization (BTEM): Application to the Homogeneous Rhodium Catalyzed Hydroformylation of 3,3-Dimethylbut-1-ene Using $\mathrm{Rh}_{4}(\mathrm{CO})_{12}$. J. Catalysis 2003, 213, 126-134.

19. Ong, L. R.; Widjaja, E.; Stanforth, R.; Garland, M. Fourier Transform Raman Spectral Reconstructions of Inorganic Lead Mixtures Using a Novel Band-Target Entropy Minimization (BTEM) Method. J. Raman Spectosc. 2003, 34, 282-289.

20. Feng, G.; Liang, Y. Z. A Novel Approach to the Retrieval of the Mass Spectrum of a Mixture. Anal. Sci. 2000, 16, 603-607.

21. Phalp, J. M.; Payne, A. W.; Windig, W. The Resolution of Mixtures Using Data from Automated Probe Mass Spectrometry. Anal. Chim. Acta 1995, 318, 43-53.

22. Garland, M.; Visser, E.; Terweisch, P.; Rippin, D.W.T. On the Number of Observable Species, Observable Reactions and Observable Fluxes in The Chemometric Studies and the Role of Multichannel Integration. Anal. Chim. Acta 1997, 351, 337358.

23. Golub, G. H.; van Loan, C. F. Matrix Computations; The Johns Hopkins University Press: Baltimore, MD, 1996, p 70.

24. Malinowski, E. R. Factor Analysis in Chemistry; Wiley: New York, NY, 1991, p 62.

25. Havrda, J. H.; Charvat, F. Quantification Methods of Classificatory Processes. Concept of Structural $\alpha$ Entropy. Kybernatica 1967, 3, 30-35.

26. Behara, M.; Chawla, J. S. Generalized $\gamma$ Entropy Selecta. Statistica Canad 1974, 2, 15-38.

27. Corana, A.; Marchesi, M.; Martini, C.; Ridella, S. Minimizing Multimodal Functions of Continuous Variables with the "Simulated Annealing" Algorithm. ACM Transactions on Mathematical Software 1987, 13, 262-282.

28. Wan, K. X.; Vidavsky, I.; Gross, M. L. Comparing Similar Spectra: From Similarity Index to Spectral Contrast Angle. J. Am. Soc. Mass. Spectrom. 2002, 13, 85-88. 